\title{
FLORA Y VEGETACIÓN ACUÁTICAS DE LOCALIDADES SELECTAS DEL ESTADO DE QUERÉTARO
}

\author{
MAHINDA MARTíneZ \\ Y \\ Adriana García Mendoza \\ Escuela de Biología \\ Universidad Autónoma de Querétaro \\ Cerro de las Campanas $\mathrm{s} / \mathrm{n}$ \\ 76010 Querétaro, Qro.
}

\begin{abstract}
RESUMEN
Se estudian la flora y vegetación acuáticas de los ríos, charcos temporales, presas y canales de riego de localidades selectas de Querétaro. En general los cuerpos de agua están contaminados y muchos están cubiertos por lirio (Eichhornia crassipes) y Lemna spp. La zona de mayor riqueza florística corresponde a los charcos temporales de la región de Amealco y Huimilpan donde se desarrollan 33 especies de plantas acuáticas y subacuáticas. En total se encontraron 118 especies de 65 géneros y 43 familias de pteridofitas, gimnospermas y angiospermas, 19 de las cuales son nuevos registros para el estado.
\end{abstract}

Palabras clave: Flora, vegetación acuática, Querétaro, México.

\begin{abstract}
The aquatic vascular flora and the vegetation of rivers, temporary ponds, dams and ditches of selected localities of the state of Querétaro, Mexico are studied. Several places are heavily infested with water hyacinth (Eichhornia crassipes) and Lemna spp. The highest floristic diversity was found in the temporary ponds of the region of Amealco and Huimilpan where 33 species of aquatic and subaquatic vascular plants were recorded. A total of 118 species of 65 genera and 43 families of pteridophytes, gymnosperms, and angiosperms were found, 19 of which are new state records for Querétaro.
\end{abstract}

Key words: Flora, aquatic vegetation, Queretaro, Mexico.

\section{INTRODUCCIÓN}

El estado de Querétaro se encuentra en el centro del país (Fig. 1) y tiene una extensión de alrededor de $11,270 \mathrm{~km}^{2}$. A pesar de ser una de las entidades más pequeñas de la República, se estima que alberga alrededor de 3,300 especies de plantas vasculares 


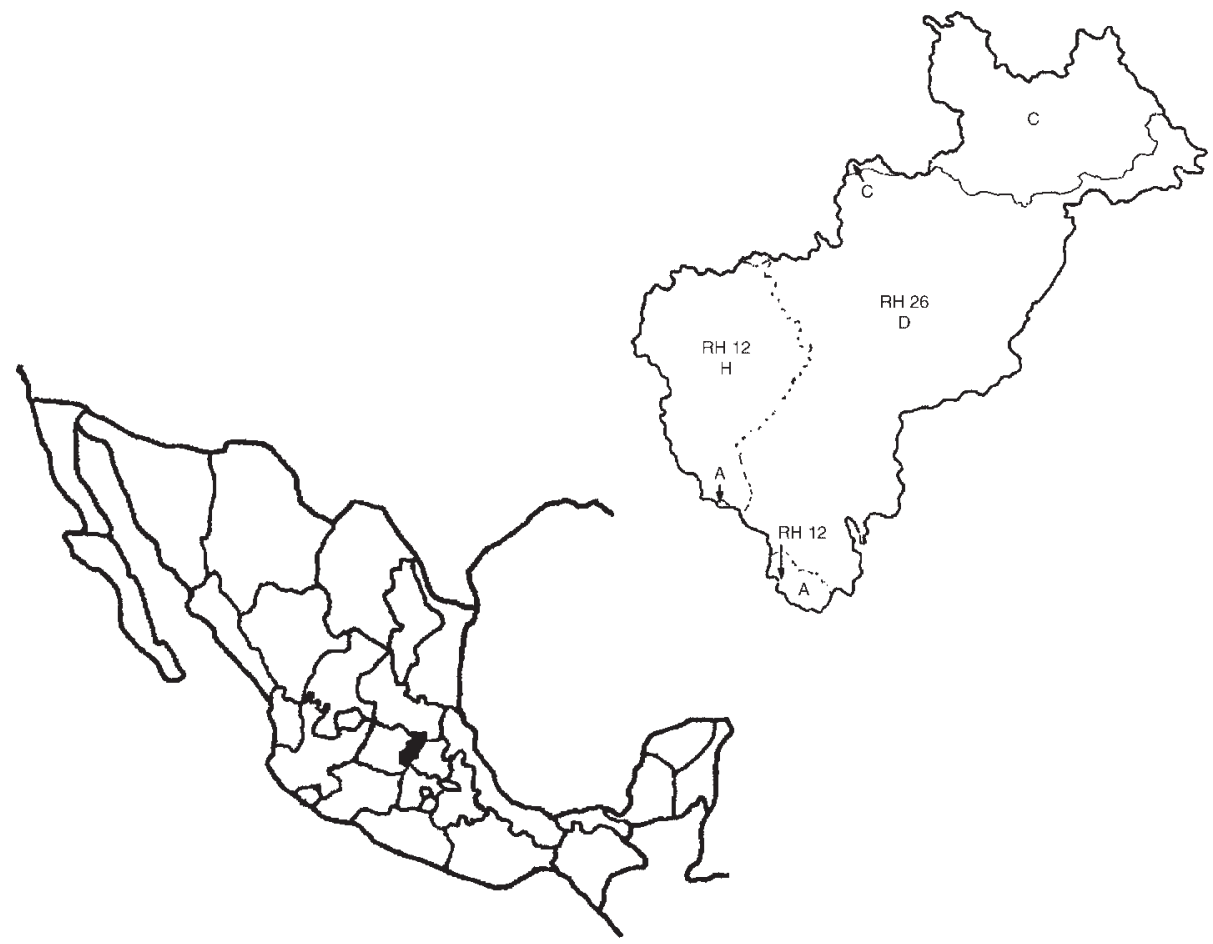

Fig. 1. Localización del estado y cuencas principales. RH 12: región hidrológica del Lerma-ChapalaSantiago; A es la cuenca del río Lerma-Toluca y H es la del río Laja. $\mathrm{RH} 26$ es la región hidrológica del Pánuco; C es la cuenca del río Tamuín y D es la cuenca del río Moctezuma. Adaptado de Anónimo (1986).

(Zamudio et al., 1992), una diversidad mayor a la de toda la península de Yucatán, Guanajuato o Coahuila.

Casi la mitad del territorio queretano está caracterizado por climas secos o semisecos, y está cubierto por una vegetación predominantemente xerófila (Anónimo, 1986; Zamudio et al., 1992). Cuenta con escasos ambientes de agua dulce, por lo que la vegetación acuática está poco desarrollada, representando un porcentaje muy bajo de la flora. Sin embargo, hay varias zonas que están bajo fuerte presión humana, con cuerpos de agua tanto temporales como permanentes que presentan plantas acuáticas y que han sido poco colectadas.

Zamudio et al. (1992) describieron la vegetación acuática en los ríos San Juan, Extórax, Moctezuma, Jalpan y Santa María; de las presas y bordos, así como la de los 
estanques naturales de Amealco y Huimilpan. Enumeraron 80 especies, algunas de las cuales se consideraron más bien como tolerantes en este trabajo.

Lot et al. $(1986,1993)$ incluyeron a Querétaro entre los ocho estados de la República con menor diversidad de plantas acuáticas, registrando únicamente 11 especies de acuáticas estrictas, aunque Argüelles et al. (1991) incluyen en su listado alrededor de 20 familias con representantes acuáticos y subacuáticos.

En la Flora del Bajío y de Regiones Adyacentes se han publicado ya los fascículos correspondientes a varias familias de plantas acuáticas o subacuáticas, entre los que se encuentran los de Carranza (1992, 1994, 1995), Calderón de Rzedowski (1996 a, b), Novelo y Ramos (1998) y Novelo y Bonilla Barbosa (1999).

En total para Querétaro se han citado en la literatura alrededor de 100 especies de plantas acuáticas y subacuáticas.

\section{METODOLOGÍA}

La delimitación de "planta acuática" es la propuesta por Lot et al. (1993). Se consideran tolerantes aquellas especies que generalmente se desarrollan en zonas secas pero que soportan suelos inundados; subacuáticas son las que completan la mayor parte de su ciclo en el borde del agua; las acuáticas estrictas son aquellas que no son capaces de sobrevivir ni siquiera por periodos cortos fuera del agua.

Las formas de vida utilizadas para las hidrófitas son las propuestas por Sculthorpe (1967), Novelo y Gallegos (1988) y Bonilla Barbosa y Novelo (1995); las hidrófitas enraizadas emergentes están ancladas al sustrato y sobresale del agua parte del tallo, las hojas y las estructuras reproductoras. Las hidrófitas enraizadas sumergidas están unidas al sustrato con las estructuras vegetativas dentro del agua, mientras que los órganos reproductores pueden estar sumergidos, emergiendo o flotando. Las hidrófitas libres flotadoras tienen tanto sus estructuras reproductoras como vegetativas sobre la superficie del agua. Las hidrófitas de hojas flotantes están ancladas al sustrato por un rizoma y generalmente tienen hojas sumergidas además de las flotadoras. Las hidrófitas de tallos postrados están enraizadas al sustrato y tienen hojas flotadoras, pero presentan tallos estoloníferos que avanzan sobre el agua. Finalmente, las hidrófitas libres sumergidas son plantas que no están unidas al sustrato, sus estructuras vegetativas están dentro del agua y los órganos reproductores pueden estar sumergidos o emergiendo. También se consideraron los árboles y arbustos que se desarrollan preferentemente en zonas inundadas y en la orilla de los ríos. Los tipos de vegetación utilizados son los propuestos por Rzedowski (1981) y Lot y Novelo (1990).

El trabajo de campo se realizó en diferentes temporadas durante dos años; se colectó en la cuenca del Lerma, los ríos El Pueblito y el de Querétaro; de la cuenca del Pánuco se exploraron los ríos Moctezuma, Escanela, Ayutla, Concá, Santa María y Extórax, así como los arroyos Zamorano y Colón en el municipio de Colón, y La Beata en el de Amealco. Se muestrearon los charcos temporales y los bordos de todo el estado. También se colectó en las presas y canales de Rayas, Santa María del Mexicano y Colón (todas del municipio de Colón), en el reservorio Constitución de 1917 y en los alrededores de La Llave (ambos en el municipio de San Juan del Río). Para la localización de los principales ríos y presas en el estado véase la Fig. 2. 


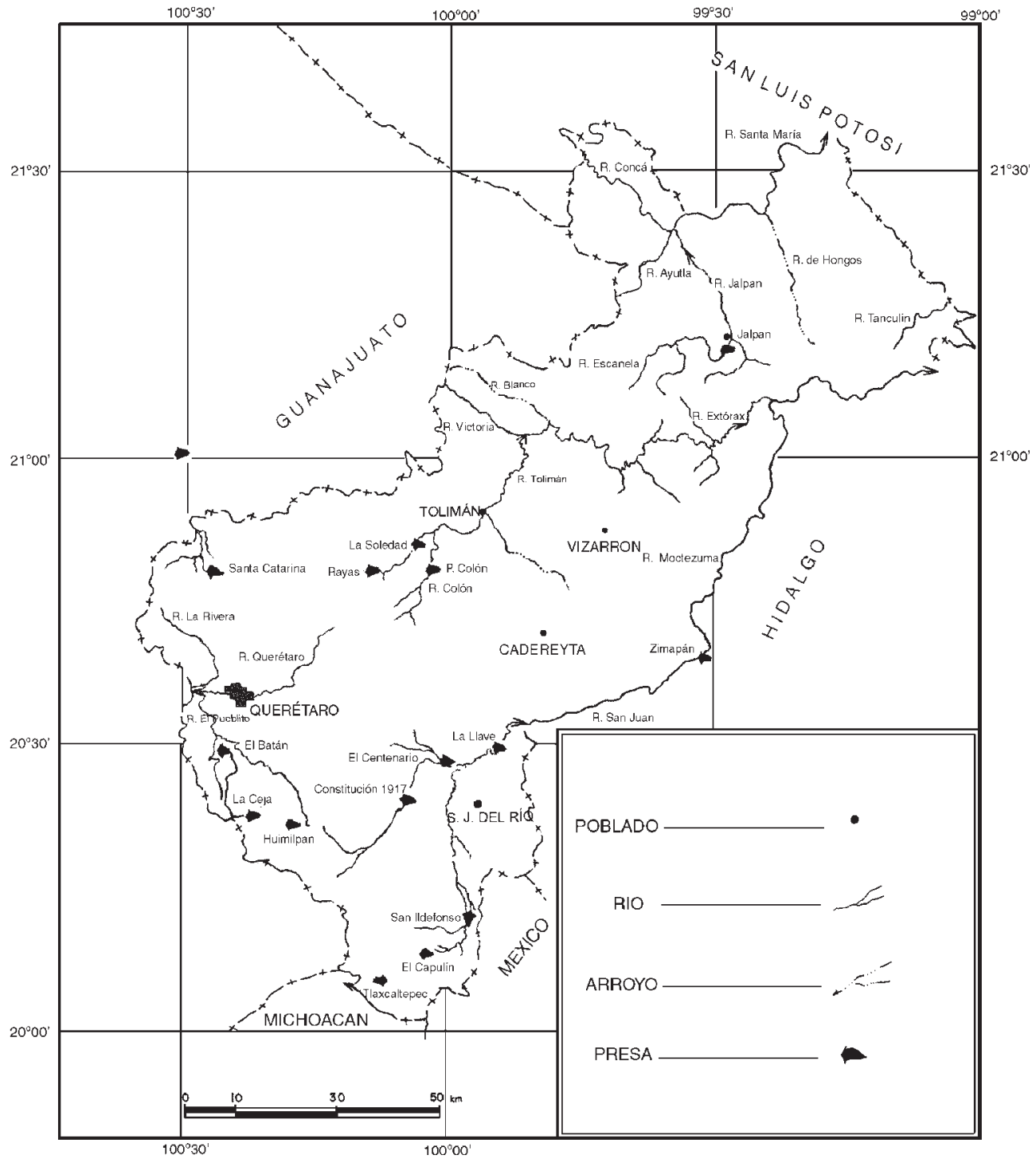

Fig. 2: Hidrología del Estado. Adaptado de Cárdenas Vargas (1992). 
En esta contribución se consideran únicamente las plantas acuáticas estrictas y las subacuáticas; se excluyen del estudio las tolerantes, aunque algunas de estas últimas también se colectaron y se enlistan en el Apéndice 2. La descripción de la vegetación se hace partiendo de la orilla hacia la parte media del cuerpo de agua. Los ejemplares colectados para este trabajo están depositados en el Herbario de la Escuela de Biología de la Universidad Autónoma de Querétaro (QMEX). Para elaborar la lista florística, se revisaron además el herbario del Centro Regional del Bajío del Instituto de Ecología (IEB) y el herbario del Instituto de Biología de la Universidad Nacional Autónoma de México (MEXU), así como los fascículos de Flora del Bajío y de regiones adyacentes y la obra de Zamudio et al. (1992). Las familias de angiospermas son las reconocidas por Cronquist (1981) y los nombres de los autores de las especies están abreviados según Brummitt y Powell (1992).

\section{DESCRIPCIÓN DEL ÁREA DE ESTUDIO}

Fisiografía: De acuerdo con Caballero (1995), el estado de Querétaro está conformado en su mayor parte por sierras y lomeríos comprendidos en tres grandes provincias fisiográficas: la Sierra Madre Oriental, la Mesa del Centro o Altiplano Mexicano y el Eje Neovolcánico Transmexicano. La Sierra Madre Oriental recorre el norte del estado y cubre $44 \%$ de su territorio. Se trata de grandes cadenas montañosas formadas por rocas marinas jurásicas y cretácicas.

La Mesa del Centro se localiza en el centro-occidente del estado y ocupa $10 \%$ de su superficie, con abundancia de llanuras y mesetas. Aunque el basamento está formado por rocas marinas del Mesozoico, éstas han sido cubiertas a partir del Terciario por rocas volcánicas.

El Eje Neovolcánico Transmexicano cubre $46 \%$ de la superficie del estado en el centro-sur, con sierras, escudos volcánicos, lomeríos, llanuras, mesetas y valles conformados por rocas de origen ígneo, sedimentos continentales del Terciario y depósitos Cuaternarios.

Climatología: Los climas que prevalecen en la Sierra Madre Oriental, en el norte del estado, son el cálido subhúmedo y el semicálido subhúmedo ambos con lluvias en verano (tipos $(\mathrm{A}) \mathrm{C}$ y $\mathrm{Aw}$ ), con 0 a 5 días con heladas en promedio anual. En el centro predominan el semiseco semicálido, semiseco templado y seco semicálido (tipo BS) con 20 a 40 días en que la temperatura desciende a $0^{\circ} \mathrm{C}$ o menos. En el sur, así como en varias áreas aisladas del resto del estado, se encuentra el clima templado subhúmedo con lluvias en verano (tipo $\mathrm{C}\left(\mathrm{w}_{2}\right)$ ) con valores medios de 20 a 60 días al año con heladas (Anónimo, 1986).

Regiones florísticas: Los patrones de distribución geográfica en el área de estudio corresponden a tres de las provincias florísticas propuestas por Rzedowski (1981), la Altiplanicie, la Sierra Madre Oriental y las Serranías Meridionales (Zamudio et al., 1992). La Altiplanicie está compuesta por una flora esencialmente xerófila con elementos de la zona árida chihuahuense y del suroeste de los Estados Unidos. La Sierra Madre Oriental presenta numerosas plantas con distribuciones que van desde Tamaulipas y Nuevo León 
hasta Hidalgo. Las Serranías Meridionales abarcan la región de Amealco, con una flora propia de las montañas del centro y sur de México. Además de estas grandes regiones, existen cañones por los que penetran elementos de la Planicie Costera del Golfo y pequeños manchones de bosque mesófilo de montaña (Zamudio et al., 1992).

Hidrología superficial: El estado está dividido en dos vertientes, la del Pacífico y la del Golfo (Fig. 1). La vertiente del Pacífico (RH 12 A y H en la Fig. 1) comprende los ríos Querétaro y El Pueblito que vierten sus aguas al río La Laja, mientras que el río Lerma capta las corrientes provenientes de los municipios de Amealco y Huimilpan (Fig. 2). Esta vertiente del Pacífico ocupa una superficie de $2,917 \mathrm{~km}^{2}$ y tiene escurrimientos anuales medios de 49 millones de $\mathrm{m}^{3}$ (Nieto, 1995).

La vertiente del Golfo de México ( $\mathrm{RH} 26 \mathrm{C}$ y $\mathrm{D}$ en la Fig. 1) ocupa la mayor parte del territorio de Querétaro y está constituida por las cuencas de los ríos San Juan, Moctezuma y Santa María, esta última con una superficie de 1,905 km². El área que drena el río Moctezuma ocupa $4,400 \mathrm{~km}^{2}$ y su afluente principal es el Extórax. El San Juan se une con el río Tula para formar el río Moctezuma y tiene una cuenca de $2,840 \mathrm{~km}^{2}$. Entre los tres presentan escurrimientos de 885 millones de $\mathrm{m}^{3}$ en promedio anual (Nieto, 1995).

Además de los ríos y arroyos, el estado cuenta con más de 70 presas para riego y generación de electricidad, las más importantes son Constitución de 1917, Centenario, Zimapán, Santa Catarina y San Pedro Huimilpan (Fig. 2, Anónimo, 1986). También hay 569 represas pequeñas o bordos para uso doméstico y control de avenidas que llegan a tener agua todo el año (Anónimo, 1986).

En los municipios de Amealco y Huimilpan existen charcos o ciénegas temporales que duran entre seis y nueve meses con agua. Algunos años se forman en mayo, otros en junio e incluso hay años en los que no se llenan. En años lluviosos hay alrededor de 40 charcos de diferentes tamaños, los más grandes tienen una superficie de 1.5 ha y los más pequeños son de alrededor de $4 \mathrm{~m}^{2}$ con profundidades promedio de $90 \mathrm{~cm}$. Se secan entre diciembre y febrero. Se localizan en lugares de escaso relieve y drenaje deficiente con sustrato de andesitas y riolitas del Terciario Superior (Zamudio et al., 1992).

En los alrededores de San Juan del Río y en varias localidades en los municipios de Colón y Pedro Escobedo se originan charcos temporales, ya sea por la presencia de pozos de extracción de agua para riego o bien por lluvias. A diferencia de los de Amealco y Huimilpan, éstos no se forman de manera regular.

\section{RESULTADOS}

Flora: Se encontraron 118 especies de plantas vasculares pertenecientes a 65 géneros y 43 familias. De estas últimas las más diversas son Cyperaceae con 20 especies, Poaceae con ocho y Apiaceae con siete. Las demás familias están representadas por seis especies (Asteraceae, Pontederiaceae) o menos.

Las plantas herbáceas hidrófitas enraizadas emergentes son las más comunes, después los árboles y en orden decreciente siguen las hidrófitas enraizadas sumergidas, las de hojas flotantes, las libres flotadoras, las de tallos postrados y finalmente las libres sumergidas y los arbustos. En el Cuadro 1 se resumen los números de especies 
correspondientes a cada forma de vida. Diecinueve de las especies encontradas son nuevos registros para el estado. (Ver listado florístico).

Cuadro 1. Número de especies acuáticas y subacuáticas de Querétaro correspondientes a diferentes formas de vida.

\begin{tabular}{|l|r|}
\hline \multicolumn{2}{|l|}{ ARBÓREAS Y ARBUSTIVAS: } \\
\hline Árboles & 10 \\
\hline Arbustos & 2 \\
\hline PLANTAS HERBÁCEAS HIDRÓFITAS: \\
\hline Enraizadas emergentes & 75 \\
\hline Enraizadas sumergidas & 9 \\
\hline Libres flotadoras & 7 \\
\hline Con hojas flotantes & 8 \\
\hline Con tallos postrados & 5 \\
\hline Libres sumergidas & 2 \\
\hline
\end{tabular}

Las plantas acuáticas colectadas en Querétaro tienen diferentes afinidades geográficas. De amplia distribución en América son 42 especies, 15 son de afinidad boreal, 13 neotropicales, 7 pantropicales, 12 cosmopolitas y 7 introducidas. Las que se comparten entre México y Guatemala son seis, de las endémicas a México se encontraron 16 y no hay ninguna restringida a Querétaro.

Principales modales de vegetación: Los tipos de vegetación acuática y asociada a ambientes de suelo húmedo que se desarrollan en Querétaro son el bosque de galería, los tulares, carrizales, vegetación flotante, vegetación sumergida y vegetación herbácea subacuática.

El bosque de galería se observa principalmente a la orilla de los ríos y las especies más importantes que lo componen son Alnus jorullensis, Fraxinus uhdei, Salix humboldtiana, Taxodium mucronatum y Platanus mexicana. Los tulares se localizan generalmente en los alrededores de las presas o en las zonas encharcadas de las carreteras y están compuestos principalmente por Typha latifolia. Los carrizales de Phragmites australis se presentan sobre todo a la orilla de ríos en los que no hay bosque de galería. La vegetación flotante compuesta por Eichhornia crassipes, Lemna gibba y Spirodela polyrrhiza puede encontrarse en las 
zonas más contaminadas como las presas o los remansos del río Moctezuma; otra asociación de plantas flotantes común es la de Azolla filiculoides, Lemna minuscula y Lemna aequinoctialis, que se desarrolla en charcos temporales de aguas limpias. La vegetación sumergida de algunos charcos está compuesta por Sagittaria demersa, Myriophyllum hippuroides, Isoëtes mexicana, Callitriche deflexa, Potamogeton spp., Utricularia perversa y Najas guadalupensis, mientras que en los ríos y arroyos se presentan Zannichellia palustris, Egeria densa y Potamogeton spp. De las plantas subacuáticas, las asociaciones más comunes están formadas por varias especies de Cyperaceae, Poaceae, Juncaceae y Apiaceae que se desarrollan ya sea a la orilla de los ríos con pocos árboles, en charcos temporales o en presas y bordos.

Vegetación de los ríos: El Pueblito (Fig. 3) tiene sectores de bosque en galería con Alnus jorullensis, Fraxinus uhdei y Salix humboldtiana, mientras que los arbustos más frecuentes son Baccharis salicifolia y Heimia salicifolia. Entre los elementos enraizados emergentes más comunes se encuentran Cyperus niger, C. pseudovegetus, Echinochloa crus-pavonis, Polygonum mexicanum, Eleocharis macrostachya, Bacopa monnieri, Typha latifolia y Mimulus glabratus y las plantas de tallos postrados están representadas por Heteranthera reniformis y Ludwigia peploides. En algunas zonas de remansos se hallan las libres flotadoras como Lemna gibba y L. aequinoctialis, mientras que del conjunto de las hidrófitas enraizadas sumergidas se observa Zannichellia palustris.

El río Querétaro está contaminado y sólo lleva agua por breves periodos después de las lluvias. La vegetación arbórea que se desarrolla en sus márgenes está compuesta por Fraxinus uhdei, Salix bonplandiana y Taxodium mucronatum, los arbustos acompañantes son Heimia salicifolia y Baccharis salicifolia. Entre las hidrófitas enraizadas emergentes crecen Cyperus odoratus, Echinochloa crus-pavonis, Heteranthera limosa, Hydrocotyle ranunculoides, Leptochloa fascicularis, Polygonum mexicanum, $P$. lapathifolium, $P$. punctatum y Rorippa nasturtium-aquaticum, de tallos postrados Ludwigia peploides y de hojas flotantes Marsilea. A veces se presenta Lemna aequinoctialis que desaparece después de las lluvias. No se encontraron plantas acuáticas sumergidas.

El río Moctezuma está altamente contaminado por las descargas de aguas negras de la ciudad de México. En sus orillas se presenta un bosque en galería de Salix humboldtiana y Taxodium mucronatum, siendo el arbusto más común Heimia salicifolia. Entre las plantas enraizadas emergentes de las orillas se desarrollan Bacopa procumbens, Hydrocotyle ranunculoides, Mimulus glabratus, Polygonum mexicanum, Ranunculus geoides y Rorippa nasturtium-aquaticum, con tallos postrados Ludwigia peploides y Heteranthera reniformis, y de hojas flotantes Marsilea. De las hidrófitas enraizadas sumergidas se encontraron Egeria densa, Potamogeton y Zannichellia palustris. Las libres flotadoras, como Eichhornia crassipes, Lemna aequinoctialis, L. gibba y Spirodela polyrrhiza son comunes en algunas zonas.

El río Escanela tiene un bosque en galería bien desarrollado de Platanus y Taxodium. Entre las plantas hidrófitas enraizadas emergentes están Bacopa procumbens, Cyperus humilis, Eleocharis geniculata, Eustoma exaltatum, Polygonum mexicanum y Ludwigia octovalvis. Como hidrófitas sumergidas se encontraron Najas guadalupensis y Potamogeton diversifolius. 
Martínez y García: Flora y Vegetación Acuáticas de Querétaro

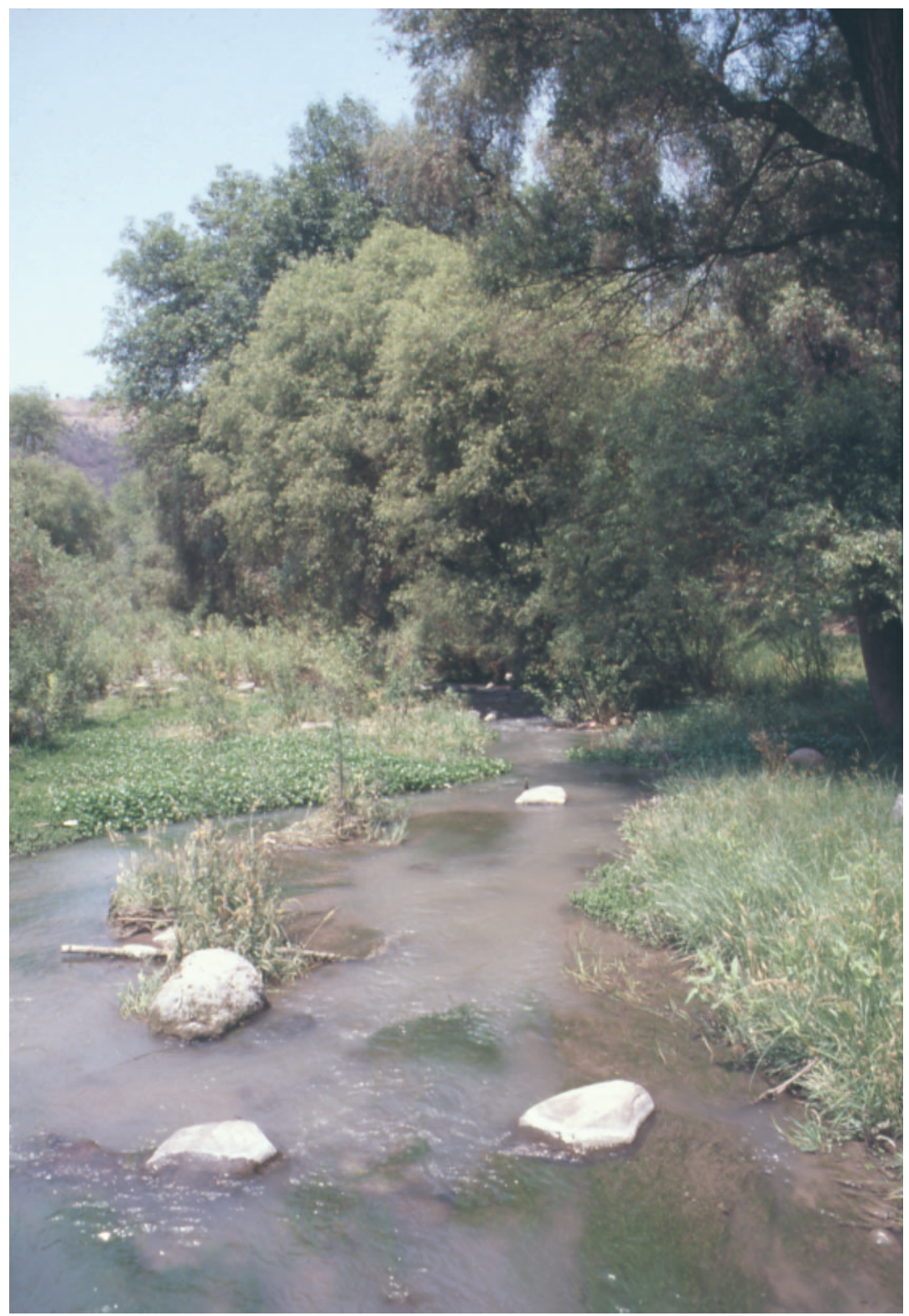

Fig. 3. Río El Pueblito con bosque en galería de Salix y Fraxinus. La sumergida es Zannichellia palustris. 
El río Ayutla también tiene árboles de Platanus, Salix y Taxodium que forman bosques de galería en sus flancos, mientras que entre las plantas herbáceas enraizadas emergentes se desarrollan Bacopa procumbens, Cyperus canus, C. humilis, Eleocharis filiculmis, E. geniculata, Samolus ebracteatus, Equisetum aff. hyemale, Polygonum aviculare y Eustoma exaltatum.

En el nacimiento del río Concá, así como en los arroyos que derivan del nacimiento y en el cauce principal, se establece Taxodium. Cyperus canus, C. odoratus, $C$. pseudovegetus, Eclipta prostrata, Hydrocotyle verticillata, Equisetum aff. hyemale, Samolus ebracteatus, Eleocharis filiculmis, E. geniculata, E. interstincta y Eustoma exaltatum son las hierbas enraizadas emergentes. La forma de tallos postrados está representada por Ludwigia peploides, la de hojas flotantes por Nymphaea ampla y Marsilea sp., mientras que de hidrófitas enraizadas sumergidas se encontró a Zannichellia palustris.

El Extórax es un río temporal en una gran parte de su recorrido. En la zona de La Higuera se desarrollan Platanus mexicana, Cyperus laevigatus, C. odoratus, C. niger, Eleocharis geniculata, Eustoma exaltatum, Polygonum sp. y Samolus ebracteatus. En el arroyo El Plátano que desemboca al Extórax se desarrollan además Cyperus niger, Eleocharis acicularis, Eustoma exaltatum, Bacopa procumbens, Marsilea sp. y Najas guadalupensis. Fuera de La Higuera y El Plátano no se encontraron plantas acuáticas o semiacuáticas en este río.

En el arroyo Zamorano y el arroyo Colón prosperan árboles de Salix, siendo los arbustos más comunes Baccharis y Heimia. Las especies enraizadas emergentes son Cyperus laevigatus, C. pseudovegetus, Eclipta prostrata, Hydrocotyle sp., Lobelia cardinalis, Mimulus glabratus, Polygonum lapathifolium, Rorippa nasturtium-aquaticum y Samolus ebracteatus. Las hidrófitas de tallos postrados están representadas por Heteranthera reniformis y Ludwigia peploides. Entre las enraizadas sumergidas se registraron Callitriche sp., Potamogeton diversifolius y Zannichellia palustris, de las libres sumergidas está presente Ceratophyllum demersum, mientras que de las libres flotadoras se encontraron Lemna aequinoctialis y Eichhornia crassipes.

En los arroyos de Amealco y Huimilpan los árboles más comunes son Alnus jorullensis, Fraxinus uhdei y Salix bonplandiana, los arbustos frecuentes son Heimia y Baccharis. De las plantas enraizadas emergentes se contaron Cyperus spp., Eleocharis macrostachya, Polygonum mexicanum, Mimulus glabratus, Juncus bufonius, Ranunculus geoides, Rorippa nasturtium-aquaticum, Tagetes persicaefolia y las hidrófitas de tallos postrados están representadas por Ludwigia peploides. Entre las enraizadas sumergidas se encontraron Potamogeton diversifolius y Zannichellia palustris y como libre flotadora a Lemna gibba.

Vegetación de los charcos temporales: Las plantas acuáticas estrictas y subacuáticas que se han encontrado en la región de Amealco y Huimilpan (Fig. 4) son las siguientes: entre las enraizadas emergentes cuentan Berula erecta, Cyperus niger, $C$. seslerioides, Elatine brachysperma, Eleocharis acicularis, E. densa, E. macrostachya, E. montana, Eriocaulon bilobatum, E. jaliscanum, Hydrocotyle ranunculoides, Lilaea scilloides, Limosella aquatica, Lythrum gracile, Polygonum mexicanum, Tagetes pringlei y Rorippa mexicana. Las hidrófitas de tallos postrados están representadas por Heteranthera peduncularis, $H$. rotundifolia, Jaegeria glabra y Ludwigia peploides. Isoëtes mexicana, 


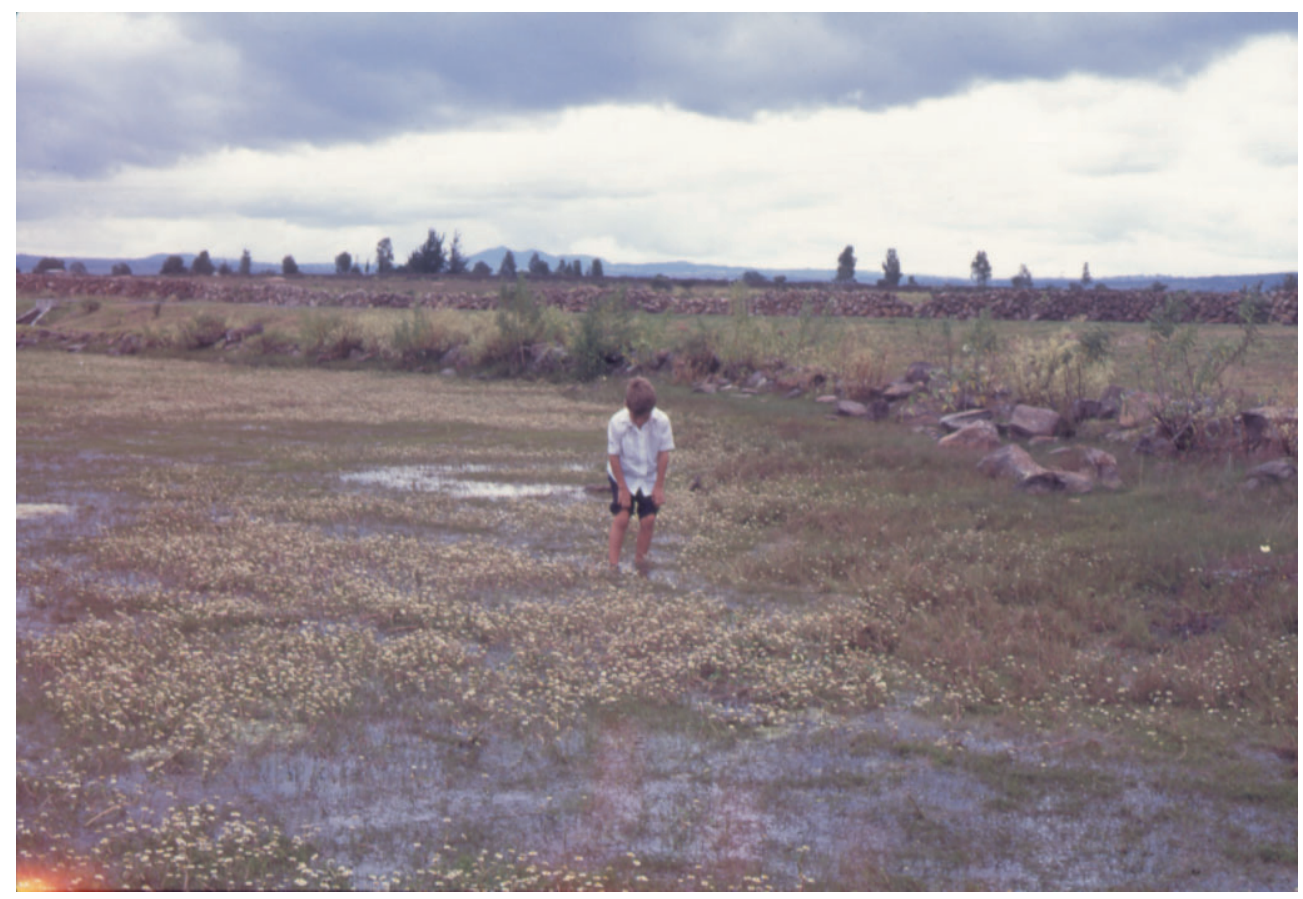

Fig. 4. Charco temporal en la zona de Amealco-Huimilpan en noviembre.

Myriophyllum hippuroides, Najas guadalupensis, Potamogeton diversifolius, Sagittaria demersa, Crassula aquatica, Zannichellia palustris son las enraizadas sumergidas y entre las de hojas flotantes se colectaron Bacopa rotundifolia, Callitriche heterophylla, Hydrochloa caroliniensis, Marsilea mollis y Nymphoides fallax. Como hidrófita libre sumergida califica Utricularia perversa y tampoco faltan las libres flotadoras Azolla filiculoides y Lemna aequinoctialis, aunque éstas últimas no se presentan todos los años y su distribución es muy localizada. A algunas de las plantas usualmente sumergidas como Sagittaria demersa - Myriophyllum hippuroides se les observan hojas fuera del agua, sobre todo hacia el final de la estación de crecimiento cuando el nivel de los embalses empieza a bajar. La riqueza florística más alta de uno de los charcos grandes llega a 31 especies de acuáticas y subacuáticas, mientras que en los cuerpos de agua menos diversos se han encontrado únicamente dos, el total correspondiente a la región de Amealco y Huimilpan suma 34 especies.

Los charcos de San Juan del Río y los del municipio de Colón tienen las siguientes especies de hidrófitas enraizadas emergentes: Cyperus niger, C. pseudovegetus, $C$. surinamensis, Datura ceratocaula, Eleocharis filiculmis, E. macrostachya, E. densa, Helenium mexicanum, Lythrum gracile, Polygonum mexicanum y Sagittaria latifolia. De las plantas con hojas flotadoras se registró a Marsilea sp., y de las de tallos postrados a 
Jaegeria glabra y Ludwigia peploides. Entre las libres flotadoras se ha encontrado de manera muy ocasional a Lemna gibba.

Vegetación de presas y canales de riego: Las presas de Colón, La Llave y Rayas presentan fuertes invasiones de Eichhornia y Typha (Fig. 5). En los canales de desagüe de los reservorios se encontró mayor diversidad de plantas acuáticas, sobre todo en el de Rayas y de Constitución de 1917, donde prospera el arbusto Heimia salicifolia y entre las hidrófitas enraizadas emergentes crecen Eleocharis sp., Hydrocotyle ranunculoides, Juncus sp., Lilaeopsis schaffneriana, Mimulus glabratus, Paspalum dilatatum, Rorippa nasturtiumaquaticum y Typha latifolia. Como planta sumergida sólo se encontró a Zannichellia palustris, y de las libres flotadoras a Azolla filiculoides, Eichhornia crassipes, Lemna gibba y Wolffia culumbiana. Las hidrofitas de tallos postrados están representadas por Heteranthera reniformis y Ludwigia peploides.

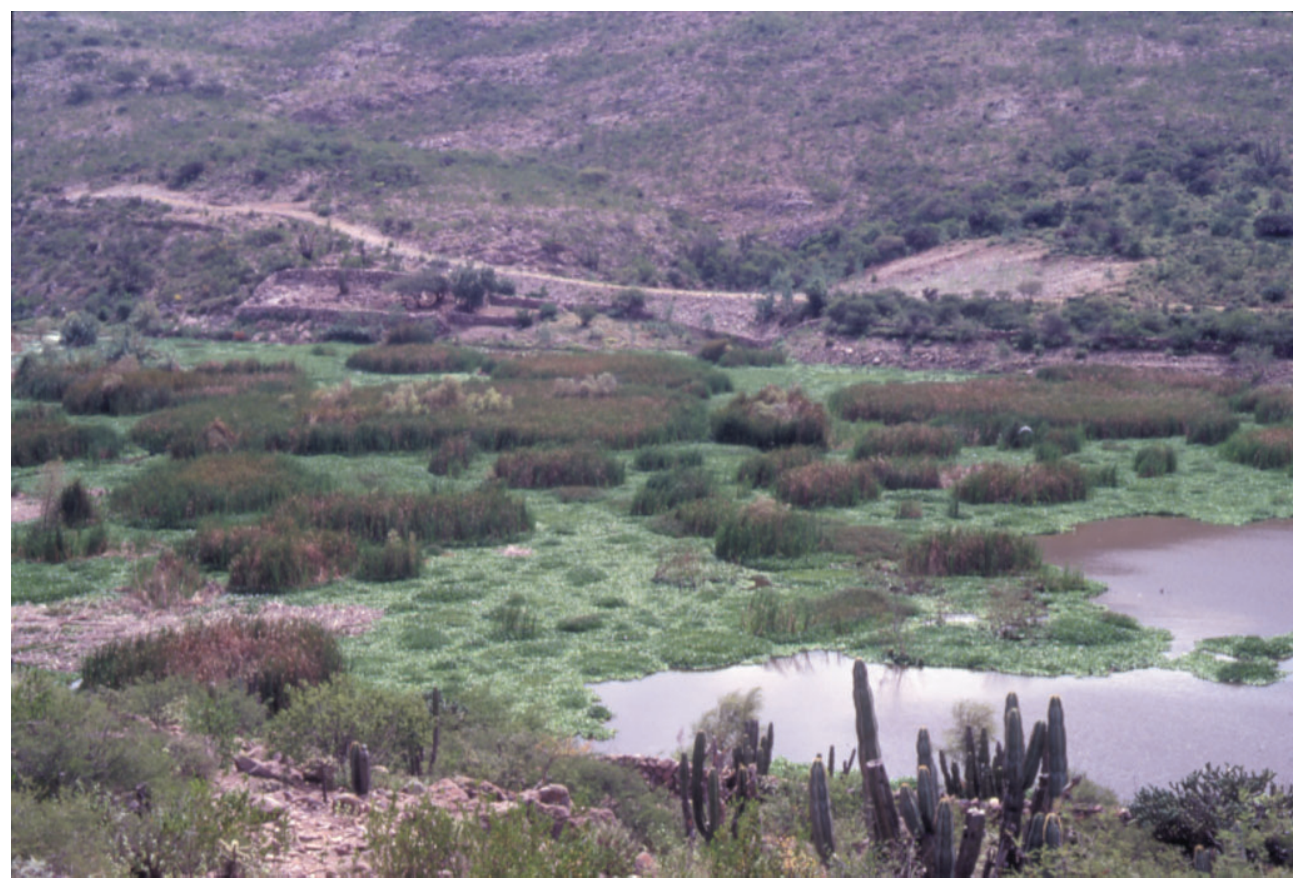

Fig. 5. Presa de la Soledad en Colón infestada por Eichhornia y Typha. 


\section{DISCUSIÓN Y CONCLUSIONES}

La flora acuática de Querétaro es más diversa de lo que se había considerado en trabajos anteriores, como se desprende de los nuevos registros obtenidos durante el desarrollo de este proyecto (ver listado florístico). Sin embargo, debido a la superficie reducida en la que se desarrolla, no es tan rica como la de otros estados, como Tamaulipas o Michoacán.

En cuanto a la afinidad florística, la mayoría de las plantas acuáticas de Querétaro son de amplia distribución en América (35.8\% del total) y las de repartición tropical (tanto neotropical como pantropical) representan 17\%. Las especies endémicas a "Megaméxico 2" de Rzedowski (1991), es decir incluyendo Centroamérica hasta el norte de Nicaragua, integran $18.8 \%$, mientras que de respetarse las fronteras políticas, la proporción desciende a $13.6 \%$. Los porcentajes más bajos los representan las especies de distribución boreal (11.9\%), las cosmopolitas (10.2\%) y las introducidas $(5.9 \%)$.

La mayor diversidad de plantas acuáticas se localiza en los charcos temporales de Amealco y Huimilpan, donde conviven hasta 31 especies en un charco, una riqueza florística mucho mayor que la que presentan varios lagos de montaña en México. Por ejemplo, de los seis lagos cráteres en Puebla, el más diverso registra siete especies y todos en conjunto apenas reúnen 14 especies, pobreza indudablemente determinada por la salinidad del agua (Ramírez y Novelo, 1984). Del Lago de Chapala en Jalisco se conocen sólo ocho especies (Borges et al., 1984 (citado por Lot y Novelo, 1988)). La diversidad de los charcos de Huimilpan - Amealco es comparable con la presentada por el lago de Yuriria (25 especies, según Ramos y Novelo, 1993) o con la de Tecocomulco que tiene 35 (Lot y Novelo, 1978). Pátzcuaro (con 48 especies, de acuerdo con Lot y Novelo, 1988) y Cuitzeo (con 55, conforme Rojas y Novelo, 1995) son más diversos, si bien son lagos de caudales permanentes y de superficies mucho mayores que los charcos de Querétaro. Los 12 que se han inventariado hasta la fecha tienen una composición florística muy similar entre sí pero substancialmente diferente de la de los lagos de montaña en México. Por ejemplo, presentan elementos como Isoëtes mexicana, Utricularia perversa, Eriocaulon bilobatum y Eriocaulon jaliscanum, ninguno de los cuales se conoce de los grandes embalses del centro del país. Otros componentes son de distribución más amplia, como Najas guadalupensis o Zannichellia palustris.

Los mencionados charcos permiten el desarrollo de 20 especies que no crecen en otras localidades del estado, y albergan a varias que ya desaparecieron del Valle de México, como Lilaea scilloides, Najas guadalupensis, Mimulus glabratus y Nymphoides fallax (Novelo y Gallegos, 1988). Infortunadamente, la riqueza de estos charcos está bajo una fuerte presión humana, ya que los cuerpos de agua están muy cercanos a las poblaciones y se usan como aguajes para los animales, por lo que los propágulos y otras estructuras de perennación a menudo se encuentran rotas o maltratadas como consecuencia del pisoteo. Varios charcos están fragmentados por caminos y carreteras y otros tienen construcciones de casas muy cercanas. Parece interesante hacer notar que los habitantes se refieren a estos charcos como "lagunas".

El estado de conservación de los cuerpos de agua de Querétaro en general es lamentable. La mayor parte de los ríos del centro y del sur están fuertemente contaminados, se llenan de Eichhornia o Lemna al menos en alguna época del año y hay algunas presas 
totalmente invadidas. Sólo las corrientes del norte en la Sierra Madre están en mejores condiciones de calidad de agua, pero son poco diversas en cuanto a las plantas acuáticas, probablemente porque están rodeadas por un bosque en galería que no permite el paso de mucha luz y a causa de la dureza del agua.

Se visitaron varias localidades muestreadas con anterioridad, y las plantas que de ahí se habían registrado ya no se encontraron. Por ejemplo, la presa de La Llave en el municipio de San Juan del Río fue visitada en 1987 por J. Rzedowski, quien colectó entre otras Sagittaria latifolia, Eleocharis macrostachya, Schoenoplectus californicus, Marsilea mollis, Potamogeton diversifolius, Echinochloa oplismenoides y Leptochloa fascicularis. Sin embargo, actualmente Eichhornia ha desplazado a todas las demás especies. Varios charcos temporales en la carretera México-Querétaro que en 1995 registraban hasta ocho especies de hidrófitas, desaparecieron recientemente con la ampliación de la carretera. Es imposible saber si estas plantas se van a establecer en otros cuerpos de agua. Sin embargo, en menos de diez años se han perdido los ambientes apropiados en varias localidades y otras se han deteriorado mucho, por lo que la diversidad de plantas acuáticas en Querétaro parece estar fuertemente amenazada.

Entre las especies que se consideran raras y vulnerables a la extinción por su distribución muy restringida se encuentran Eriocaulon bilobatum, Eriocaulon jaliscanum (Calderón de Rzedowski, 1996a), Heteranthera mexicana (Novelo y Ramos, 1998), Lobelia cardinalis (Rzedowski y Calderón de Rzedowski, 1997), Nymphaea ampla (Novelo y Bonilla Barbosa, 1999) y Anemopsis californica (Calderón de Rzedowski, 1996b). A éstas se pueden añadir otras que se conocen sólo de un charco, como Myriophyllum hippuroides, Elatine brachysperma y Bacopa rotundifolia.

\section{AGRADECIMIENTOS}

Este trabajo se llevó a cabo gracias a la ayuda económica de la Comisión Nacional para el Conocimiento y Uso de la Biodiversidad bajo el proyecto H076 "Flora acuática de Querétaro". La colecta en la zona de Amealco y Huimilpan se ha continuado con el apoyo del Consejo Nacional de Ciencia y Tecnología bajo el proyecto 29104N. Maricela Gómez S. determinó las gramíneas, Nelly Diego las ciperáceas, Alejandro Novelo algunas acuáticas estrictas y Ma. de la Luz Arreguín algunos helechos. Jerzy Rzedowski y Graciela Calderón de Rzedowski amablemente nos acompañaron al campo y nos mostraron las localidades más interesantes. Alejandro Novelo y Luis Hernández revisaron el manuscrito y nos acompañaron al campo. Jacinto Treviño y Gilberto Ocampo auxiliaron en algunas etapas del proyecto. Dos revisores anónimos aportaron sugerencias para mejorar la versión final.

\section{LITERATURA CITADA}

Anónimo, 1986. Síntesis geográfica del estado de Querétaro. Instituto Nacional de Informática, Geografía. México, D.F. 143 pp.

Argüelles, E., R. Fernández y S. Zamudio. 1991. Listado florístico preliminar del estado de Querétaro. Instituto de Ecología A. C. Flora del Bajío y de Regiones Adyacentes. Fascículo complementario II. Pátzcuaro, Mich. 155 pp. 
Bonilla Barbosa, J. y A. Novelo. 1995. Manual de identificación de plantas acuáticas del Parque Nacional Lagunas de Zempoala, México. Cuadernos 26, Instituto de Biología. Universidad Nacional Autónoma de México. México, D.F. 168 pp.

Borges, S. A., M. Gómez-Higareda, A. Gutiérrez, M. Hinojosa y O. Villarreal. 1984. Macrófitas acuáticas en el lago de Chapala, Jal. Servicio Social Universidad Autónoma Metropolitana, Iztapalapa. México, D.F. 83 pp.

Brummitt, R. K. y C. E. Powell. 1992. Authors of plant names. Royal Botanic Gardens. Kew. 732 pp.

Caballero, J. A. 1995. Fisiografía. Geografía de Querétaro. Universidad Autónoma de Querétaro y Academia Queretana de Estudios Humanísticos A.C. Querétaro, Qro. 385 pp.

Calderón de Rzedowski, G. 1996a. Eriocaulaceae. Flora del Bajío y de Regiones Adyacentes. Instituto de Ecología A.C. Fascículo 46. Pátzcuaro, Mich. 11 pp.

Calderón de Rzedowski, G. 1996b. Saururaceae. Flora del Bajío y de Regiones Adyacentes. Instituto de Ecología A.C. Fascículo 42. Pátzcuaro, Mich. 5 pp.

Cárdenas Vargas, J. 1992. Geological-mining monograph of the state of Querétaro. Consejo de Recursos Minerales. México, D.F. 108 pp.

Carranza, E. 1992. Taxodiaceae. Flora del Bajío y de Regiones Adyacentes. Instituto de Ecología A.C. Fascículo 4. Pátzcuaro, Mich. 7 pp.

Carranza, E. 1994. Platanaceae. Flora del Bajío y de Regiones Adyacentes. Instituto de Ecología A.C. Fascículo 23. Pátzcuaro, Mich. 6 pp.

Carranza, E. 1995. Salicaceae. Flora del Bajío y de Regiones Adyacentes. Instituto de Ecología A.C. Fascículo 37. Pátzcuaro, Mich. 21 pp.

Cronquist, A. 1981. An integrated system of classification of flowering plants. Columbia University Press. Nueva York. 262 pp.

Lot, A. y A. Novelo. 1978. Laguna de Tecocomulco, Hidalgo. Guías botánicas de excursiones en México. Sociedad Botánica de México. México, D.F. 19 pp.

Lot, A. y A. Novelo. 1988. Vegetación y flora acuática del Lago de Pátzcuaro, Michoacán, México. Southw. Natur. 33(2): 167-175.

Lot, A. y A. Novelo. 1990. Forested wetlands of Mexico. In: Lugo, A.E., M.M. Brinson y S. Brown (eds.) Ecosystems of the World. Forested wetlands of the World. Elsevier Publ. Co. Amsterdam. pp. 287-298.

Lot, A., A. Novelo y P. Ramírez G. 1986. Listados florísticos de México V. Angiospermas acuáticas mexicanas. Instituto de Biología, Universidad Nacional Autónoma de México, D.F. 60 pp.

Lot, A., A. Novelo y P. Ramírez G. 1993. Diversity of Mexican aquatic vascular plant flora. In: Ramamoorthy, T. P., R. Bye y J. Fa (eds.). Biological diversity of Mexico: origins and distribution. Oxford University Press. Nueva York. pp 557-591.

Nieto, J. 1995. Hidrografía. Geografía de Querétaro. Universidad Autónoma de Querétaro y Academia Queretana de Estudios Humanísticos A.C. Querétaro, Qro. 385 pp.

Novelo, A. y J. Bonilla Barbosa. 1999. Nymphaeaceae. Flora del Bajío y de Regiones Adyacentes. Instituto de Ecología A.C. Fascículo 77. Pátzcuaro, Mich. 11 pp.

Novelo, A. y L. Ramos. 1998. Pontederiaceae. Flora del Bajío y de Regiones Adyacentes. Instituto de Ecología A.C. Fascículo 63. Pátzcuaro, Mich. 19 pp.

Novelo, A. y M. Gallegos. 1988. Estudio de la flora y la vegetación acuática relacionada con el sistema de chinampas en el sureste del Valle de México. Biotica 13: 121-140.

Ramírez G. P. y A. Novelo. 1984. La vegetación acuática vascular de seis lagos-cráter del estado de Puebla, México. Bol. Soc. Bot. México 46: 75-88.

Ramos, L. J. y A. Novelo. 1993. Vegetación y flora acuática de la Laguna de Yuriria, Guanajuato, México. Acta Bot. Mex. 25: 61-79.

Rojas M., J. y A. Novelo. 1995. Flora y vegetación acuáticas del Lago de Cuitzeo, Michoacán, México. Acta Bot. Mex. 31: 1-17. 
Rzedowski, J. 1981. Vegetación de México. Ed. Limusa. México, D.F. 432 pp.

Rzedowski, J. 1991. El endemismo en la flora fanerogámica mexicana: una apreciación preliminar. Acta Bot. Mex. 15: 47-64.

Rzedowski, J. y G. Calderón de Rzedowski. 1997. Campanulaceae. Flora del Bajío y de Regiones Adyacentes. Instituto de Ecología A.C. Fascículo 58. Pátzcuaro, Mich. 64 pp.

Sculthorpe, C. D. 1967. The biology of aquatic vascular plants. Edward Arnold Publ. Londres. 610 pp.

Zamudio, S., J. Rzedowski, E. Carranza y G. Calderón de Rzedowski. 1992. La vegetación en el estado de Querétaro, panorama preliminar. Instituto de Ecología A.C., México. pp. 75-78.

Recibido en mayo de 1999. Aceptado en diciembre de 2000. 


\title{
APÉNDICE 1
}

Lista florística: Las formas de vida están indicadas por las siguientes abreviaturas: A - árbol. Ar arbusto. EE - enraizada emergente. ES - enraizada sumergida. HF - hojas flotantes. LF - libre flotadora. LS - libre sumergida. TP - tallos postrados. El asterisco denota nuevos registros para el estado, es decir que no estaban citados en Argüelles et al. (1991), Zamudio et al. (1992) o los fascículos de Flora del Bajío.

\author{
ALISMATACEAE \\ Sagittaria demersa J. G. Sm., ES-EE \\ S. latifolia Willd., EE \\ S. longiloba Engelm. ex Torr., EE
}

\section{APIACEAE}

Berula erecta Coville, EE

Eryngium cervantesii Delar., EE

Hydrocotyle mexicana Schltdl. \& Cham., EE

$H$. ranunculoides L. f., EE

H. umbellata L., EE

$H$. verticillata L., EE

* Lilaeopsis schaffneriana (Schltdl.) Coult. \& Rose, EE

\section{ASTERACEAE}

Baccharis salicifolia (Ruiz \& Pavón) Pers., Ar

Eclipta prostrata L., EE

Helenium mexicanum Kunth, EE

Jaegeria glabra B. L. Rob., TP

J. hirta (Lag.) Less., EE

Tagetes pringlei S. Watson, EE

\section{AZOLLACEAE}

${ }^{*}$ Azolla filiculoides Lam., LF

\section{BETULACEAE}

Alnus acuminata Kunth, A

A. jorullensis Kunth, A

\section{BRASSICACEAE}

Rorippa mexicana (Moc. \& Sessé) Standl. \& Steyerm., EE

R. nasturtium-aquaticum (L.) Hayek, EE

\section{CALLITRICHACEAE}

* Callitriche deflexa A. Braun ex Hegelm. ES

C. heterophylla Pursh, HF

\author{
CAMPANULACEAE \\ Lobelia cardinalis L., EE \\ CERATOPHYLLACEAE \\ Ceratophyllum demersum L., LS
}


Apéndice 1. Continuación.

CRASSULACEAE

Crassula aquatica Maxim., EE

CYPERACEAE

C. ochraceus Vahl, EE

C. canus Presl, EE

C. flavescens L., EE

C. humilis Kunth, EE

*C. laevigatus L., EE

C. niger Ruiz \& Pavón, EE

C. odoratus L., EE

* C. pseudovegetus Steud., EE

C. surinamensis Rottb., EE

Eleocharis acicularis (L.) Roem. \& Schult., EE

E. bonariensis Nees, EE

* E. densa Benth., EE

* E. filiculmis Kunth, EE

E. geniculata (L.) Roem. \& Schult., EE

* E. interstincta (Vahl) Roem. \& Schult., EE

E. macrostachya Britton, EE

* E. montana (Kunth) Roem. \& Schult., EE

*E. montevidensis Kunth, EE

Fuirena simplex Vahl, EE

Schoenoplectus californicus (C. Meyer) Soják, EE

ELATERIACEAE

Elatine brachysperma A. Gray

EQUISETACEAE

Equisetum aff. hyemale L., EE

ERIOCAULACEAE

Eriocaulon bilobatum Morong, EE

E. jaliscanum S. Watson, EE

GENTIANACEAE

Eustoma exaltatum (L.) Salisb., EE

HALORAGACEAE

* Myriophyllum hippuroides Nutt. ex Torr. \& A. Gray, ES

HYDROCHARITACEAE

Egeria densa Planch., ES

ISOËTACEAE

Isoëtes mexicana Underw., ES-EE 
Apéndice 1. Continuación.

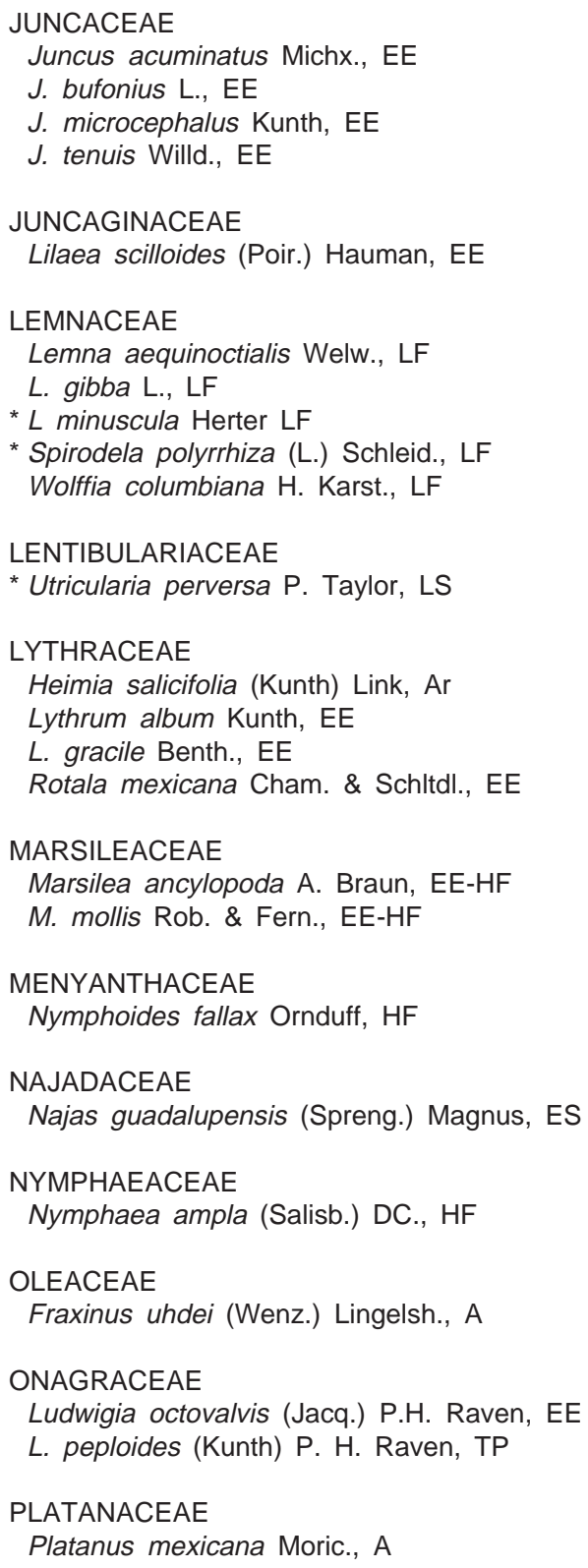


Apéndice 1. Continuación.

POACEAE

* Echinochloa crus-pavonis (Kunth) Schult., EE E. oplismenoides Hitchc., EE

* Glyceria fluitans (L.) R. Br., HF Hydrochloa caroliniensis P. Beauv., HF Leptochloa fascicularis (Lam.) A. Gray, EE

* Paspalum dilatatum Poir., EE $P$. distichum L., EE

Phragmites australis (Cav.) Trin. ex Steudel, EE

POLYGONACEAE

Polygonum hydropiperoides Michx., EE

P. lapathifolium L., EE

P. mexicanum Small, EE

P. punctatum Ell., EE

\section{PONTEDERIACEAE}

Eichhornia crassipes (C. Mart.) Solms, LF

Heteranthera limosa (Sw.) Willd., EE

H. mexicana S. Watson, EE

$H$. peduncularis Benth., TP

H. reniformis Ruiz \& Pav., TP

H. rotundifolia (Kunth) Griseb., TP

\section{POTAMOGETONACEAE}

Potamogeton diversifolius Raf., ES-HF

$P$. foliosus Raf., ES

$P$. nodosus Poiret, ES-HF

$P$. pectinatus L., ES

PRIMULACEAE

Samolus ebracteatus Kunth, EE

RANUNCULACEAE

Ranunculus hydrocharoides A. Gray, EE

R. geoides Kunth, EE

\section{SALICACEAE}

Salix bonplandiana Kunth, A

S. humboldtiana Willd., A

S. paradoxa Kunth, A

S. schaffneri Schn., A

S. taxifolia Kunth, A

\section{SAURURACEAE}

Anemopsis californica (Nutt.) Hook. \& Arn., EE 
Apéndice 1. Continuación.

SCROPHULARIACEAE

Bacopa monnieri (L.) Wettst., EE

B. procumbens (Mill.) Greenm., EE

${ }^{*} B$. rotundifolia (Michx.) Wettst., HF Limosella aquatica L., EE

Mimulus glabratus Kunth, EE

SOLANACEAE

Datura ceratocaula Ort., EE

TAXODIACEAE

Taxodium mucronatum Tenn., A

TYPHACEAE

Typha latifolia L., EE

ZANNICHELIACEAE

Zannichellia palustris L., ES 
Acta Botanica Mexicana (2001), 54: 1-23

APÉNDICE 2

Lista florística de especies tolerantes.

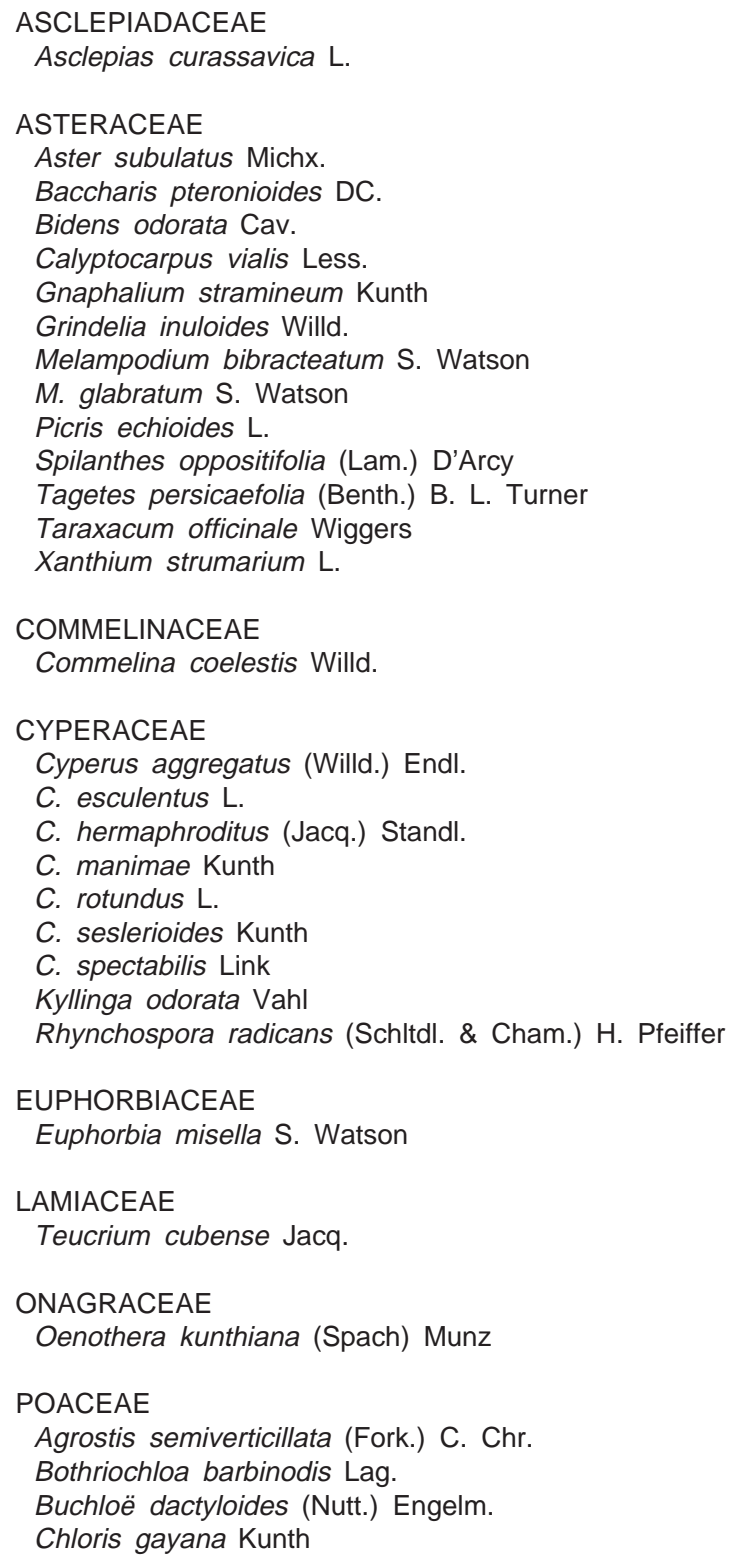


Apéndice 2. Continuación.

Cynodon dactylon (L.) Pers.

Echinochloa colonum (L.) Link

E. crusgalli (L.) Beauv.

Leptochloa dubia (Kunth) Nees

Lolium multiflorum Lam.

Panicum hians Ell.

$P$. vaseyanum Scribn.

Paspalum distichum $\mathrm{L}$.

$P$. lividum Trin.

$P$. notatum Flügge

$P$. tenellum Willd.

Polypogon elongatus Kunth

P. monspeliensis (L.) Desf.

$P$. viridis (Gouan) Breistr.

Setaria geniculata (Lam.) Beauv.

Sporobolus indicus (L.) R. Br.

PLANTAGINACEAE

Plantago major L.

\section{POLYGONACEAE}

Polygonum aviculare $\mathrm{L}$.

Rumex crispus L.

R. mexicanus Meisn.

PRIMULACEAE

Anagallis arvensis $\mathrm{L}$.

SCROPHULARIACEAE

Capraria biflora L.

SOLANACEAE

Nicotiana plumbaginifolia Viviani 